\title{
Maximising the hybrid effect in unidirectional hybrid composites
}

\author{
Yentl Swolfs* ${ }^{1}$, Ignaas Verpoest ${ }^{1}$, Larissa Gorbatikh ${ }^{1}$ \\ ${ }^{1}$ Department of Materials Engineering, KU Leuven, \\ Kasteelpark Arenberg 44 bus 2450, Belgium \\ *Corresponding author: yentl.swolfs@mtm.kuleuven.be
}

\begin{abstract}
The failure strain of fibre-reinforced composites can be increased by fibre hybridisation. A recently developed model for unidirectional composites was extended to hybrid composites to analyse this synergetic effect, called the hybrid effect. The model predicts individual fibre breaks and interactions among clusters of fibre breaks. Three key parameters were studied to understand how they can maximise the hybrid effect, namely low elongation fibre strength scatter and hybridisation fibre stiffness and failure strain. Larger strength scatter of the low elongation fibres leads to larger hybrid effects, as the scatter spreads out the cluster development over a larger strain interval. The failure strain ratio of the two fibre types should be above 2 for the properties used here, but a higher ratio did not yield any additional benefits. Increasing the stiffness of the hybridisation fibre reduces the stress concentrations on the low elongation fibre and may also enlarge the hybrid effect. These conclusions provide guidelines for designing optimal hybrid composites.
\end{abstract}

Keywords: Carbon fibres; Hybrid composites; Polymer-matrix composites (PMCs); Probabilistic methods; Hybrid effect; Tensile failure.

\section{Graphical abstract}
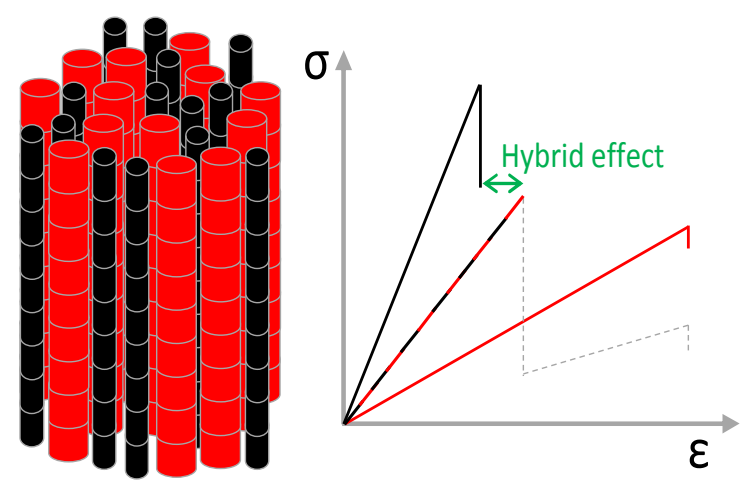

\section{Highlights}

- A longitudinal strength model for unidirectional hybrid composites incorporating realistic fibre packings was developed.

- The apparent failure strain increase of the carbon fibres, the so-called hybrid effect, was investigated.

- Larger strength scatter of the low elongation fibres leads to strong increases in the hybrid effect.

- The hybrid effect initially increases with increasing failure strain of the high elongation fibre, but then levels off.

- A stiffer higher elongation fibre can slightly increase the hybrid effect. 


\section{Introduction}

Fibre-hybridisation is one of the few strategies that can apparently increase the failure strain of carbon fibre composites, while still exploiting the excellent mechanical properties of carbon fibres. In fibre-hybridisation, a hybridisation fibre is added to the low elongation fibres, often with the aim of achieving a better balance in performance. Carbon fibres are typically hybridised with glass [1-9] and aramid [10-13] fibres, but other fibres have also been used: flax [14], polyethylene [15] and polypropylene [16] fibres.

Hayashi [6] was the first to report an apparent failure strain increase of carbon fibres. Hayashi found that when carbon fibre layers were sandwiched in between glass fibre layers, the failure strain of the carbon fibre layers was $40 \%$ higher than for a composite consisting of only carbon fibre layers. This increase is called the 'hybrid effect'. It is often termed an apparent increase, as the intrinsic failure strain or strength of the carbon fibres does not change. Other authors have later reported similar increases $[3,13,17,18]$.

A recent review paper [19] summarised the three hypotheses for the hybrid effect that have been coined so far: (a) residual thermal stresses [8,17], (b) dynamic stress concentrations [20], and (c) failure development [13,21]. The first two hypotheses certainly play a role in the hybrid effect, but can only account for a small part $[8,13,17,20]$. The third hypothesis, namely failure development, requires an understanding of the failure of unidirectional (UD) non-hybrid composites. Failure of these composites begins with individual fibre breaks. Those fibres locally stop carrying load and shed their load to the adjacent fibres, which causes stress concentrations on the adjacent fibres [22-27]. The broken fibre recovers its load due to shear stress transfer in the matrix. This effect decreases the stress concentrations on the adjacent fibres as a function of the axial distance from the break. Nevertheless, the stress concentrations increase the failure probability of the adjacent fibres, which causes the tendency to develop clusters of fibre breaks [28-32]. These clusters further intensify the stress concentrations. Finally, a critical cluster develops which propagates in an unstable manner and causes final failure of the composite. Adding a hybridisation fibre can significantly alter the failure development and thereby increase the apparent failure strain of the low elongation fibres in the hybrid composite $[3,13,18,19,21,33,34]$.

Many parameters exist that can help to increase the hybrid effect, but most of them are relatively poorly understood in literature. These include the fibre dispersion, the relative volume fraction of both fibres, the low elongation fibre strength scatter, the failure strain ratio and the fibre stiffness ratio. Well dispersed fibres and small low elongation fibre fractions are known to maximise the hybrid effect. This has been extensively demonstrated in experiments $[3,19]$, and now also recently in models [21]. The importance of the other three parameters however has not been quantified yet. Firstly, the importance of the low elongation fibre strength scatter has been identified by several researchers [13,33,35]. A larger strength scatter or lower Weibull modulus is thought to yield a higher hybrid effect, but quantitative predictions are lacking.

Secondly, Zweben's model indicated that the failure strain ratio of both fibre types is crucial for the hybrid effect [13]. As proven by Fukuda [33], this was caused by Zweben's approximate definition of the hybrid effect. Fukuda's model improved this definition and found no influence of the failure strain ratio. Zweben and Fukuda laid the ground work for modelling of hybrid composites. Unfortunately, both authors used simple 1D packings, consisting of a single row of fibres, and did not model the entire failure development. Consequently, their models can only give indications of certain trends, but cannot be used to draw strong 
conclusions. More refined models are needed to establish the importance of the failure strain ratio for maximising the hybrid effect.

Finally, it is well recognised that the stiffness of both fibre types affects the stress concentrations around a fibre break in hybrid composites [13,33,34]. Its importance for the hybrid effect however, has not been quantified yet.

This study extends an existing strength model for UD composites to UD hybrid composites. The focus lies on carbon/glass hybrid composites, but these fibres will be referred to as low elongation (LE) and high elongation (HE) fibres respectively to keep the descriptions generic. The model for hybrid composites in Swolfs et al. [21] ignored the difference in fibre radiuses and used a simplified stress redistribution around the fibre breaks based on very local load sharing. Nevertheless, it was successful in predicting experimentally measured hybrid effects [36]. The model used here is the very first study to include the difference in fibre radiuses and uses randomly dispersed fibres in a random fibre packing. This is essential as regular packings would cause the larger fibres to be closer to the broken fibre than the smaller fibres. In a carbon/glass hybrid composite, this would lead to a too pronounced shielding effect and hence to overestimations of the hybrid effect. Additionally, geometrical limitations would restrict the overall fibre volume fraction in regular packings.

Three key parameters for maximising the hybrid effect are analysed: the LE fibre strength scatter, the failure strain ratio, and the stiffness of the HE fibre. The influence of hybrid volume fraction and dispersion will not be analysed here, as we have already analysed these parameters in detail earlier [21]. The focus is on the hybrid effect for the initial failure strain, which corresponds to propagation of the first critical cluster. It is not the intention to predict what happens afterwards, as that is often a complicated mix of fragmentation, fibre bundle debonding, delamination growth and more fibre breaks. The stiffness and strength will not be discussed here, but the stiffness can easily be obtained from the linear rule-of-mixtures. The strength would follow directly from the stiffness and failure strain, as all our predictions are linearly elastic.

\section{Model description}

\subsection{General approach}

The general approach of the model has been extensively described in literature $[21,23,32,37]$ and is similar to the works of Curtin, Okabe and co-workers [24,38-40]. The model starts off by creating a representative volume element (RVE) consisting of parallel fibres. These fibres are divided into fibre elements, as illustrated in Fig. 1. A strength consistent with a Weibull distribution is assigned to each element. The fibre bundle consists of a random fibre packing of two fibre types, which are randomly dispersed and have a different radius (see Fig. 1).

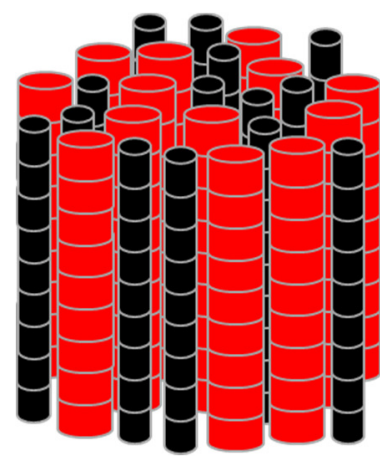

Figure 1: Illustration of the chain-of-bundles approach for LE/HE hybrid composites. Each fibre is divided into fibre elements. The image is not to scale and the actual model contains more and longer fibres. 
The model gradually increments the global strain and checks for element failure by comparing element strength to element stress. If no new element fails in this strain increment, then the strain is further incremented. If new elements have failed, then the model recalculates the stress redistribution around these broken elements. The model first searches for clusters of fibre breaks. Two fibre breaks are considered to be part of a cluster if (1) the lateral distance between their fibre centres is less than 4 LE fibre radiuses, and (2) the axial distance between the fibre breaks is less than 10 LE fibre radiuses. Fibre breaks that are further away have a negligible influence on each other [22,34]. The definition of a cluster was the same irrespective of the fibre properties and the applied stress level.

The model then updates the stress concentration factors. This update is performed by first assuming that there are no interactions among fibre breaks and then correcting for those interactions. The stress concentration factors (SCFs) without interactions are obtained from the stress fields in finite element (FE) models for hybrid composites with a single fibre break. The FE model takes into account the radius of both fibre types, but assumes a linear elastic and well-bonded matrix. Using an elastic matrix is an approximation that will affect the predicted failure strains. Including plasticity would increase or decrease the failure strains of the reference and hybrid composite in a similar way. The influence on the hybrid effect will therefore be minor, as it is defined as the relative ratio of these two failure strains. Including plasticity would however require the difficult implementation of an ineffective length that changes with applied strain. An alternative approach would be to use the ineffective length at a strain that is close to the failure strain. Given that the hybrid effect will change the failure strain, this may introduce additional and unwanted artefacts. Due to these reasons, the best solution was to use an elastic matrix.

A tiny matrix crack was added to avoid a stress singularity in the broken fibre. An extensive description can be found in Swolfs et al. [23,34]. The SCFs were defined as the relative increase compared to the nominal stress level. The ineffective length was defined as the relative distance from the break at which $90 \%$ of the nominal fibre stress is recovered.

The stress profiles from the FE solutions are imported into the strength model using trend line equations for various characteristic points. Interactions among fibre breaks are taken into account through an enhanced superposition principle. This principle has been described and validated in Swolfs et al. [23]. It is based on linear superposition of the individual fibre break solutions with a correction to satisfy force equilibrium.

After updating the SCFs, the model calculates element stresses and checks for element failure again. This entire procedure is repeated until the failure criterion is satisfied, after which the model is interrupted. The failure criterion is satisfied if more than $10 \%$ of the fibres are broken within an axial segment of $35 \mu \mathrm{m}$, which corresponds to the length of $10 \mathrm{LE}$ fibre elements. This criterion corresponds to a large number of iterations within a single strain increment. This indicates that a critical cluster is growing unstably, and further computation would become slow and meaningless.

The development of clusters as well as global strain $\varepsilon$ and average composite stress $\sigma_{c}$ are tracked at each strain increment. The composite stress is calculated according to equation (1), which is the sum of three components. The first component is the sum of all element stresses $\sigma_{e l}$ divided by the number of elements $n_{e l}$ and multiplied by the fibre volume fraction $V_{f}$. The second component is calculated by multiplying the matrix tensile modulus $E_{m}$ by the applied strain $\varepsilon$ and the matrix volume fraction $V_{m}$. The final component $\sigma_{S C F} . \mathrm{V}_{m}$ is small 
and comes from the additional matrix stresses caused by the fibre breaks. The value of $\sigma_{m, S C F}$ was derived from the FE models with a single fibre break [22,34].

$$
\sigma_{c}=\frac{\sum \sigma_{e l}}{n_{e l}} V_{f}+\left(E_{m} \varepsilon+\sigma_{m, S C F}\right) V_{m}
$$

In accordance with Swolfs et al. [19], the hybrid effect was calculated as the relative failure strain increase of the LE fibres in the hybrid composite compared to that of the all-LE fibre composite. It should be emphasised that the failure strain of the hybrid composite corresponds to the development of a critical cluster of LE fibre breaks. The model does not attempt to model what happens afterwards.

\subsection{Model parameters}

The model parameters are summarised in Table 1. The cylindrical model contains 2000 fibres plus an additional 250 fibres on the perimeter. These boundary fibres are added to avoid preferential cluster formation at the perimeter [37]. They are given an infinite strength to prevent their failure and are not taken into account in the average composite stress. The length of the RVE is $10 \mathrm{~mm}$, which corresponds to 2857 elements/fibre having a length of $3.5 \mu \mathrm{m}$. A total of 50 simulations are performed for each configuration.

The fibres inside the RVE are arranged in a random fibre packing to maximise the randomness in the hybrid composites. The packings are created by a random fibre packing generator, which was developed by Melro et al. [41] and validated by Romanov et al. [42]. The generator was adapted for the purpose of this study to allow two different fibre radiuses [34]. The overall fibre volume fraction as well as the hybrid volume fraction were fixed at $50 \%$. The hybrid volume fraction is defined as the relative volume fraction of HE fibres over all fibres.

The default properties of the HE fibre are chosen to be similar to that of a typical glass fibre to facilitate a comparison with literature. The fibre radiuses for LE and HE fibre were $3.5 \mu \mathrm{m}$ and $6 \mu \mathrm{m}$ respectively, which are typically values for carbon and glass fibres, respectively. Their longitudinal stiffnesses are assumed to be 230 and $70 \mathrm{GPa}$ respectively, compared to $3 \mathrm{GPa}$ for the matrix. The anisotropy of the carbon-like LE fibre is not used in the strength model itself, but is taken into account through the SCFs obtained from FE simulations [22]. The HE fibre and matrix are assumed to be isotropic.

A modified Weibull distribution $P$ was used:

$$
P=1-\exp \left(-\left[\frac{L}{L_{0}}\right]^{\alpha}\left[\frac{\sigma_{f}}{\sigma_{0}}\right]^{m}\right),
$$

where $L$ is the element length, $L_{0}$ is the characteristic gauge length, $\sigma_{f}$ is the fibre strength, $\sigma_{0}$ is the Weibull scale parameter and $m$ is the Weibull shape parameter or Weibull modulus. The exponent $\alpha$ is a correction factor that accounts for the gauge length-dependency of the standard Weibull distribution. While there is considerable discussion on this gauge length dependency [43-45], using these parameters facilitates comparisons with a simpler model [46]. The Weibull parameters for the LE fibres are $\sigma_{0}=4493 \mathrm{MPa}, L_{0}=10 \mathrm{~mm}, \mathrm{~m}=4.8$ and $\alpha=0.6$. For HE fibres, the default parameters are taken from a glass fibre data set: $\sigma_{0}=1550 \mathrm{MPa}$, 
$L_{0}=24 m m, m=6.34$ and $\alpha=1$ [47]. Some of these default properties will be varied to analyse their effect.

Table 1: Summary of the default model parameters.

$\begin{array}{lc}\text { Number of regular fibres } & 2000 \\ \text { Number of boundary fibres } & 250 \\ \text { Model length } & 10 \mathrm{~mm} \\ \text { Number of elements/fibre } & 2857 \\ \text { Element length } & 3.5 \mu \mathrm{m} \\ \text { Number of simulations per configuration } & 50 \\ \text { Overall fibre volume fraction } & 50 \% \\ \text { LE fibre radius } & 3.5 \mu \mathrm{m} \\ \text { HE fibre radius } & 6 \mu \mathrm{m} \\ \text { Longitudinal LE fibre stiffness } & 230 \mathrm{GPa} \\ \text { Longitudinal HE fibre stiffness } & 70 \mathrm{GPa} \\ \text { Matrix stiffness } & 3 \mathrm{GPa} \\ & 4.8 \text { (LE fibre) } \\ \text { Weibull modulus } m & 6.34 \text { (HE fibre) } \\ \text { Weibull scale parameter } \sigma_{0} & 4493 \mathrm{MPa} \text { (LE fibre) } \\ & 1550 \mathrm{MPa} \text { (HE fibre) } \\ \text { Factor } \alpha & 0.6 \text { (LE fibre) } \\ \text { Weibull reference gauge length } L_{0} & 1 \text { (HE fibre) } \\ \end{array}$

\section{Results}

\subsection{Fibre strength scatter}

Zweben [13] developed the first model for hybrid composites. Zweben's seminal work pointed towards the Weibull modulus of LE fibre as one of the key parameters for maximising the hybrid effect. Assessing the importance of this parameter is, however, not straightforward. By changing just the Weibull modulus of the LE fibres, the failure strain of the all-LE fibre composite also changes. To maintain a constant reference for calculating the hybrid effect, this failure strain should remain the same. This can be achieved by simultaneously changing the Weibull scale parameter $\sigma_{0}$. The Weibull moduli are chosen to be 3, 4, 4.8, 6 and 10. The corresponding Weibull scale parameters $4285 \mathrm{MPa}, 4411 \mathrm{MPa}, 4493 \mathrm{MPa}, 4580 \mathrm{MPa}$ and $4710 \mathrm{MPa}$, respectively, were obtained by running 200 simulations, calculating the average failure strain and adjusting $\sigma_{0}$. After 2-3 iterations, all scale parameters yielded failure strains for the all-LE fibre composite that were statistically indistinguishable from $1.75 \%$.

The hybrid effects for 50/50 LE/HE hybrids with varying LE fibre Weibull moduli are summarised in Fig. 2. This clearly illustrates the importance of the Weibull modulus of LE fibre for the hybrid effect. A hybrid effect of $7 \%$ is expected for the actual Weibull modulus of 4.8. By decreasing this to 3, the hybrid effect is nearly doubled. By increasing this to 10 , which indicates a very small strength scatter, the hybrid effect decreases to $1 \%$. 


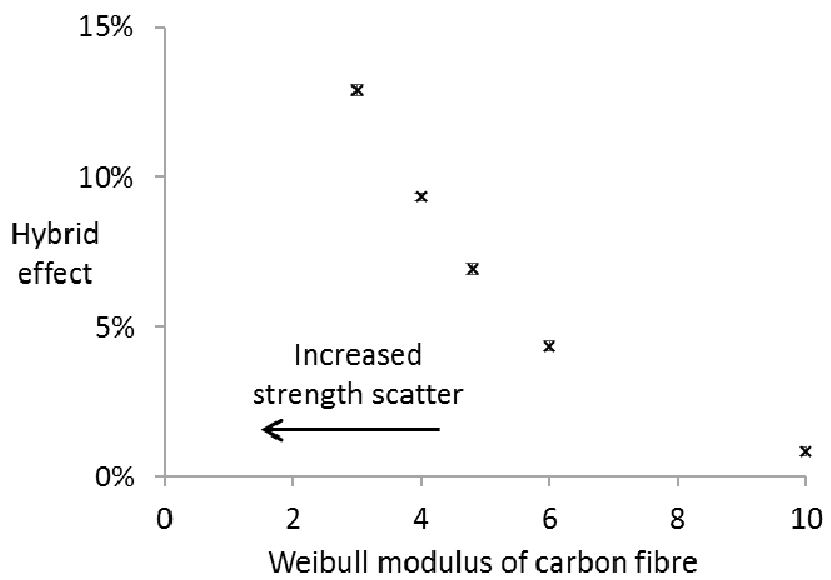

Figure 2: The hybrid effect as a function of the Weibull modulus. The five all-LE fibre composites all had a failure strain of $1.75 \%$.

While the predicted hybrid effects may not be very large, one should keep in mind the following arguments:

- The hybrid effects will be larger when the LE fraction is lower than $50 \%$ [3,19,21].

- The new carbon fibres that are being developed for the automotive sector are likely to have a larger strength scatter, and therefore more potential for larger hybrid effects.

- While the addition of glass fibres will decrease the stiffness by less than half, the cost will decrease by nearly a factor of two. This can lead to significant cost savings.

Fig. 3 plots the evolution of 3-plets in the reference composites and the corresponding hybrid composites for the five different Weibull moduli. The full lines indicate the reference composites, which intentionally all have the same failure strain. The dashed lines indicate the corresponding hybrid composites. All hybrid composites have a delayed cluster development compared to their reference composites. This delay becomes smaller for higher Weibull moduli, as the development of 3-plets occurs over a much smaller strain interval. Fibrehybridisation is therefore an efficient way to delay cluster development, and the delay is less pronounced for high Weibull moduli.

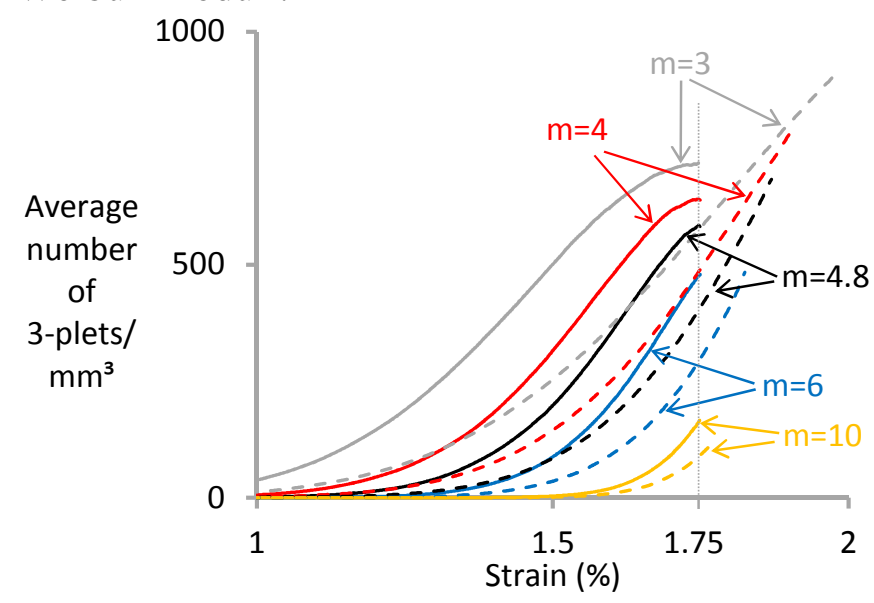

Figure 3: The evolution of 3-plets/ $\mathrm{mm}^{3}$ as a function of the applied strain. Full lines indicate the reference all-LE fibre composites, while dashed lines indicate the corresponding 50/50 LE/HE hybrid composites. The all-LE fibre composites all have a failure strain of $1.75 \%$, as indicated by the vertical line. The results were averaged over 50 simulations. 
The results in Fig. 3 illustrate that large hybrid effects in carbon/glass hybrid composites were easier to achieve in the seventies, when carbon fibres were not only weaker, but also had a lower Weibull modulus [48]. Maximising the hybrid effect may hence be easier in some carbon fibre types. Firstly, T300 carbon fibres are known to have a lower Weibull modulus than T700 fibres and therefore may have a larger potential for hybrid effects. Secondly, pitch-based carbon fibres are known to have a lower Weibull modulus [49] and hence have more potential for large hybrid effects.

\subsection{HE failure strain}

The importance of the HE failure strain has been debated in literature. In literature, this often referred to using the failure strain ratio, which is the ratio of the failure strain of the HE fibre over that of the LE fibre. Zweben's model indicated a larger ratio would strongly increase the hybrid effect [13], while Fukuda later did not find any influence [33]. While both models were crucial for the initial understanding of hybrid composites, they are based on simple 1D fibre packings with a 50/50 ratio of both fibre types. This can introduce significant artefacts and drastically limit the accuracy of both models. Their definitions of the hybrid effect are based on failure of the nearest (an HE fibre) or second nearest (an LE fibre) neighbour, which does not necessarily mean that the failure will propagate.

In the present work, the failure strain of the HE fibre composite was varied by increasing the Weibull scale parameter $\sigma_{0}$ of the HE fibre while keeping the other parameters constant. This means that the HE fibre has a $70 \mathrm{GPa}$ stiffness in all cases. The failure strain ratio was defined as the ratio of the failure strain of the all-HE fibre composite over that of the all-LE fibre composite. In the previous section with a Weibull modulus of 6.34 and 4.8 for the HE and LE fibres, the obtained reference failure strains were $3.51 \%$ and $1.75 \%$ respectively. This led to a failure strain ratio of 2 . It should be emphasised that the failure strain ratio of the composites was used and not that of the fibres.

Fig. 4 plots the hybrid effect for 50/50 LE/HE hybrids with various failure strain ratios. These ratios could for example be achieved if carbon fibre was hybridised with natural fibres or with oriented polymer fibres. A strong increase is seen up to a failure strain ratio of about 2 , after which the hybrid effect levels off. This leads to two vital conclusions. Firstly, to maximise the hybrid effect, the failure strains of both fibre types should be sufficiently far apart. Secondly, adding more ductile fibres while maintaining the same fibre stiffness does not lead to a larger hybrid effect. This means that the importance of the failure strain ratio was overestimated by Zweben [13], but underestimated by Fukuda [33].

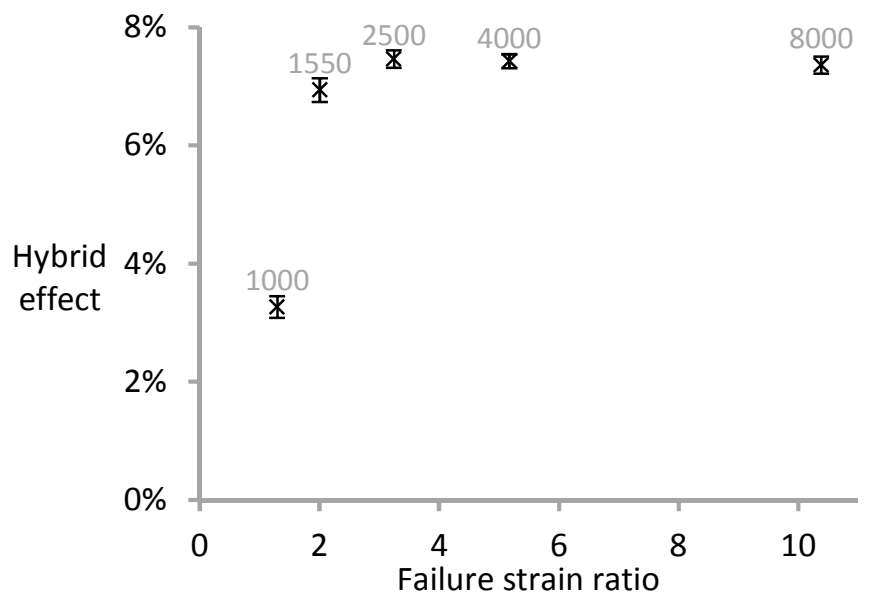

Figure 4: The hybrid effect for 50/50 LE/HE hybrid composites at various failure strain ratios. The data labels indicate the Weibull scale parameter $\sigma_{0}$ of the HE fibre. 
The question arises why a failure strain ratio above 2 does not lead to an increased hybrid effect. A first hint can be found in Fukuda's work on 1D packings with alternating LE/HE fibres [33]. Fukuda assumes that a hybrid composite fails when the LE fibre breaks that is the second nearest neighbour to an already broken LE fibre. Fukuda implicitly assumes that the nearest neighbour, which is an HE fibre, does not fail. In that case, the failure strain ratio can be expected to have no influence on the hybrid effect. This reasoning can be extended to the current model. The failure probability of the HE fibres is not zero as in Fukuda's model, but is relatively low compared to that of LE fibre.

To confirm this hypothesis, Fig. 5 plots the average number of HE fibre breaks as a function of applied strain. The number of HE fibre breaks is indeed small compared to the 20.000-40.000 LE fibre breaks near final failure. The curve for a failure strain ratio of 10 is not even plotted because the number of HE fibres was extremely low. Higher failure strain ratios delay the onset of HE fibre breaks and drastically reduce the number of HE fibre breaks near final failure. Nevertheless, failure strain ratios of 2 and higher still lead to hybrid composite with the same failure strain. The occurrence of HE fibre breaks is hence not needed, as a cluster of only LE fibre breaks can also develop into a critical one. This explains why the hybrid effect levels off for failure strain ratios above 2 .

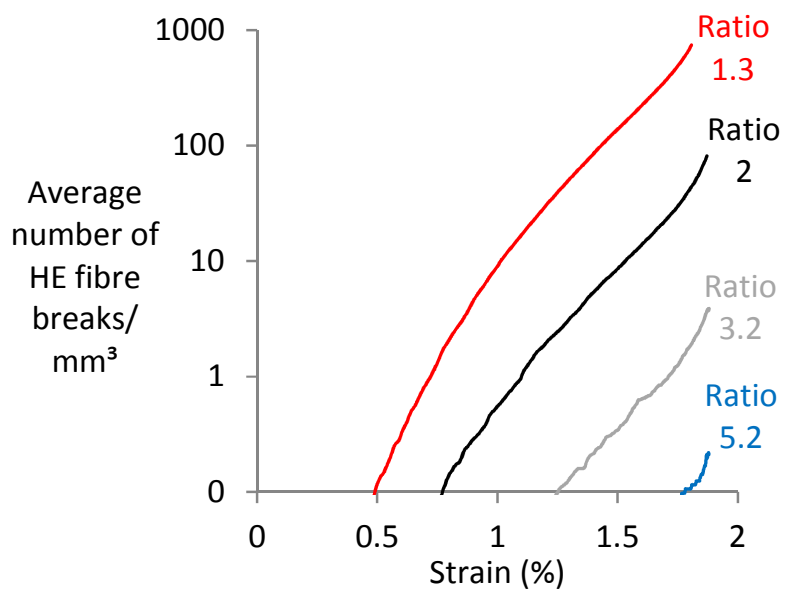

Figure 5: Average number of $\mathrm{HE}$ fibre breaks $/ \mathrm{mm}^{3}$ for hybrid composites with different failure strain ratios. The results were averaged over 50 simulations.

These conclusions have three limitations. Firstly, the model assumes final failure when the critical cluster develops in the LE fibres. This critical cluster is detected when the number of fibre breaks increases exponentially within the same strain increment. At that point, most $\mathrm{HE}$ fibres are still intact. Especially at high failure strain ratios, the HE fibres should be able to continue carrying load. The model is currently not able to predict this residual load carrying capacity. This would however only change the ultimate failure strain. The hybrid effect itself would not change, however, as its definition is based on the delay of the failure of the LE fibres in a hybrid composite.

Secondly, the model highlighted a failure strain ratio of 2 as the threshold value for maximising the hybrid effect. This threshold is expected to depend on several material parameters, of which the Weibull moduli of both fibre types is likely to be the most important one. These moduli determine the overlap in the failure strain of both fibres and are thereby important in controlling the hybrid effect.

Finally, the previous results assume that the failure strain increases 10-fold, while the fibre maintains the same tensile modulus. In practice, however, an increased failure strain is often 
associated with more compliant fibres. More compliant fibres are less efficient load carriers, which should also affect the hybrid effect. The influence of the HE fibre stiffness will be investigated in more detail in the next section.

\subsection{HE fibre stiffness}

The influence of the longitudinal fibre stiffness on the stress redistribution was analysed by changing the HE fibre stiffness from $70 \mathrm{GPa}$ to $40 \mathrm{GPa}$ and $10 \mathrm{GPa}$ in the FE models. Such stiffness values are common in several natural fibres (such as hemp, bamboo and jute) as well as in oriented polymer fibres (such as polypropylene and polyethylene naphthalate). The Poisson's ratio of the HE fibre was kept constant at 0.22 , which implies that the shear stiffness decreases together with the longitudinal HE fibre stiffness. All other parameters and HE composite strength in particular were kept the same. This implies that the average failure strain of the HE fibres increased proportionally with the decreasing HE fibre stiffness. Because the failure strain ratio of two is exceeded, this increased HE failure strain will not affect the outcome of the predictions.

The stiffness decrease drastically increases the SCFs on the intact HE fibres (see Fig. 6a). It should be kept in mind that the SCF is defined as a relative stress increase compared to the nominal stress level. Since the lower HE stiffness reduces this nominal stress level, a small absolute stress increase actually causes a large SCF on the HE fibres. In general, however, reducing the longitudinal stiffness of the HE fibre reduces its load carrying capacity. This causes a slight increase in the SCFs on the intact LE fibres with decreasing HE fibre stiffness (see Fig. 6b).

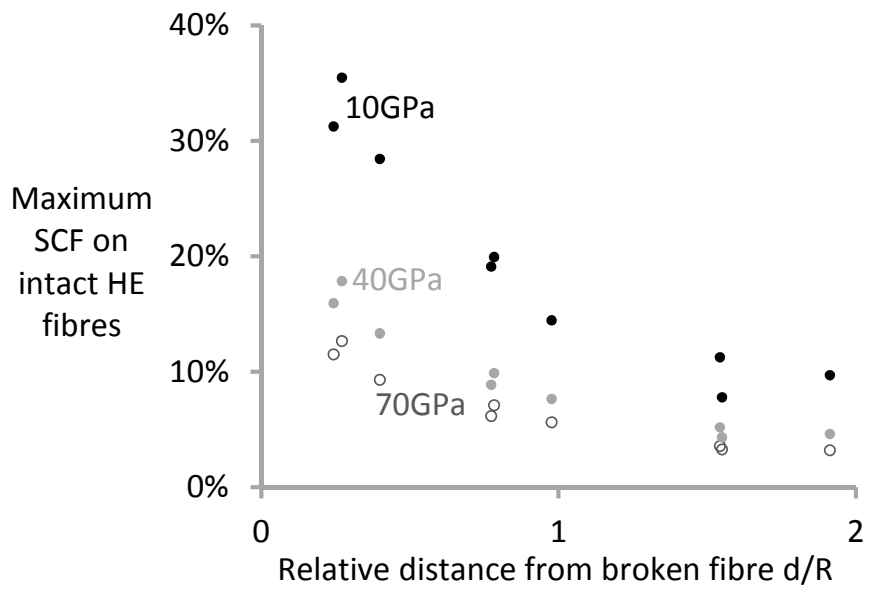

(a)

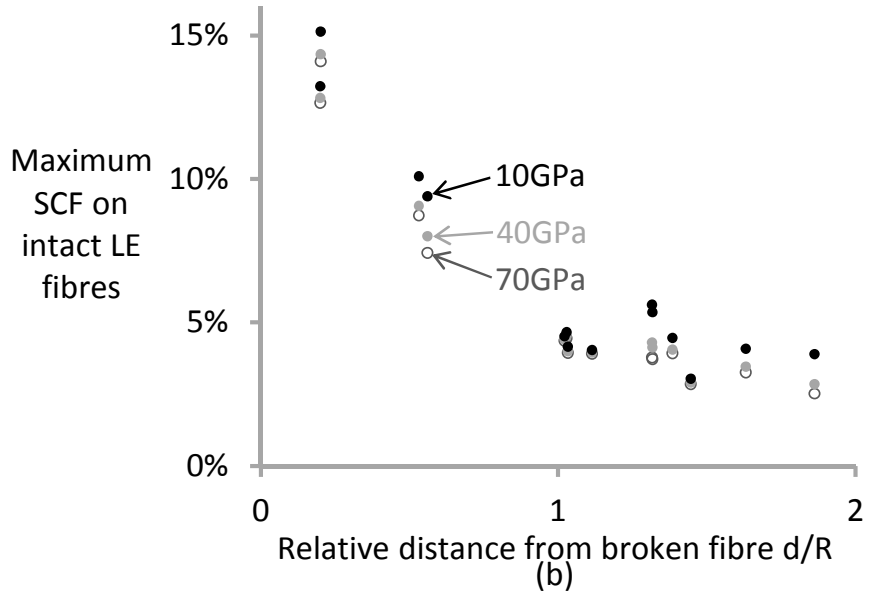

Figure 6: The maximum stress concentration factors around a broken LE fibre for HE fibres with a stiffness of 10, 40 and 70 GPa: (a) for intact HE fibres, and (b) for intact LE fibres. The results of 5 FE models are plotted for each HE fibre stiffness. The hybrid and overall volume fraction was $50 \%$ in all cases. 
The ineffective length of a broken LE fibre seems to slightly increase with decreased HE fibre stiffness (see Fig. 7). This increase is however not statistically significant. Such a small increase can be expected from theoretical considerations. The shear stiffness of the intact fibres contributes to the stress recovery of the broken fibre [22,34]. By reducing the HE fibre stiffness, its shear stiffness is also reduced. This should lead to a slower stress recovery in the broken fibre and hence a longer ineffective length.

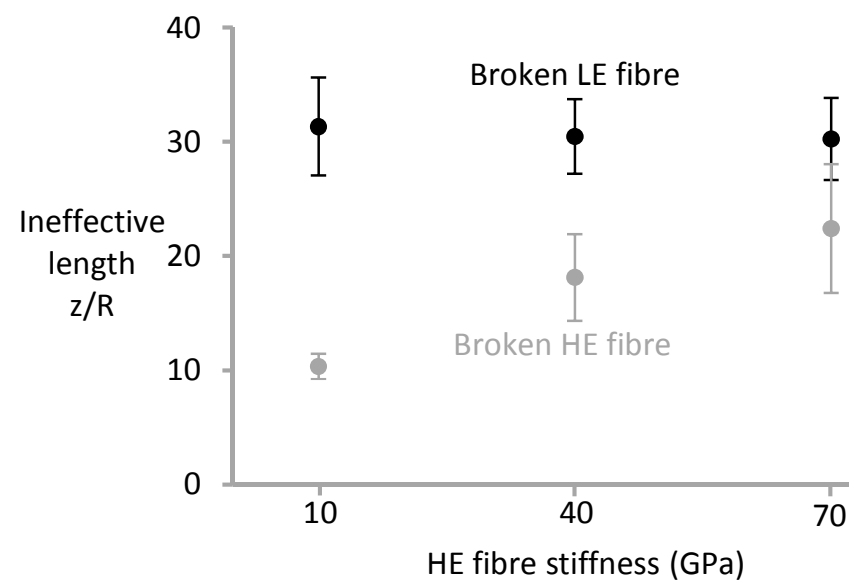

Figure 7: The ineffective length for broken $\mathrm{LE}$ and $\mathrm{HE}$ fibres as a function of the HE fibre stiffness. The overall $V_{f}$ was $50 \%$.

In contrast, the ineffective length of a broken HE fibre strongly reduces with decreasing $\mathrm{HE}$ fibre stiffness. A low HE fibre stiffness requires less stress build up to reach the nominal value again. The stress recovery hence occurs faster, resulting in a shorter ineffective length for a low HE fibre stiffness.

The data in Fig. 6 and Fig. 7 were entered into the strength model, while all the other parameters were kept constant. The hybrid effect for 50/50 hybrid LE/HE composites was predicted. By keeping $\sigma_{0}$ constant, a decreasing HE fibre stiffness will increase the failure strain of the all-HE fibre composite. As shown previously, this failure strain ratio has a negligible influence on the hybrid effect, as the HE failure strain ratio is 2 or higher.

A decreased HE fibre stiffness slightly decreases the hybrid effect (see Fig. 8). This decrease is attributed to the slight increase of the stress concentrations on the LE fibres (see Fig. 6).

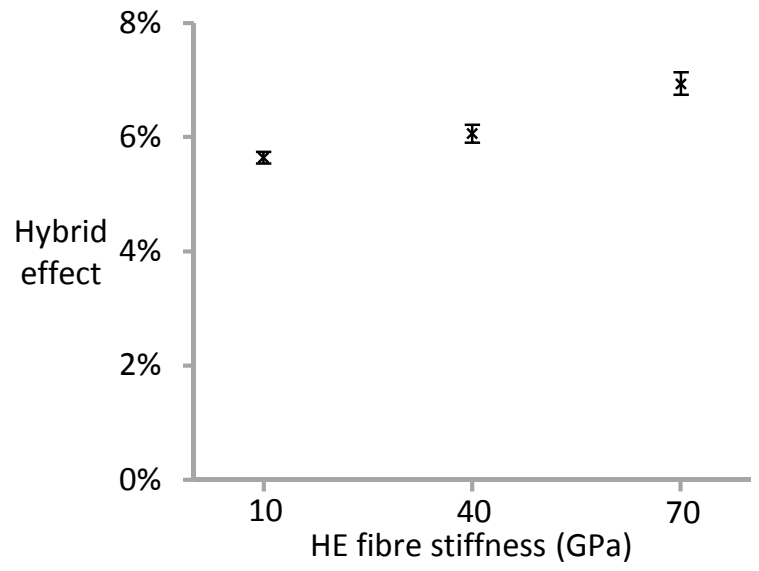

Figure 8: The effect of HE fibre stiffness on the hybrid effect in 50/50 LE/HE hybrid composites. The overall $V_{f}$ was $50 \%$. 


\section{Conclusions}

An existing strength model was extended to model UD hybrid composites. A major improvement compared to state-of-the-art models is that the present model uses a fully random dispersion of LE and HE fibres with their actual fibre radius. The model was used to analyse three key parameters for hybrid composites: LE fibre strength scatter, failure strain ratio and HE fibre stiffness. If all other parameters are assumed to be constant, then the hybrid effect can be maximised in three ways:

- A large LE fibre strength scatter (or small Weibull modulus) leads to earlier development of clusters, which facilitates the delay of this development by fibre hybridisation.

- A sufficiently high failure strain ratio should be aimed for. With the properties used here, the ratio should be above 2, but a higher ratio did not lead to additional benefits.

- A high fibre stiffness helps to reduce the stress concentrations on the LE fibres.

It should be noted that these three parameters cannot always be changed independent of each other. For example, the strength scatter and failure strain of fibres are intrinsically linked together by the presence of flaws.

A further parametric study is required to further optimise the hybrid effect. The effect of some parameters, such as matrix stiffness or fibre-matrix debonding, is still unknown in literature. This model has been previously compared with experimental data for non-hybrid composites [32] and a simplified version has been compared to experimentally measured hybrid effects [36]. This validation will be performed in the near future.

\section{Acknowledgements}

The work leading to this publication has received funding from the European Union Seventh Framework Programme (FP7/2007-2013) under the topic NMP-2009-2.5-1, as part of the project HIVOCOMP (Grant Agreement No. 246389). The authors thank the Agency for Innovation by Science and Technology in Flanders (IWT) for the grant of Y. Swolfs. I. Verpoest holds the Toray Chair in Composite Materials at KU Leuven.

\section{References}

[1] Jalalvand M, Czél G, Wisnom MR. Numerical modelling of the damage modes in UD thin carbon/glass hybrid laminates. Composites Science and Technology. 2014;94:39-47.

[2] Czél G, Wisnom MR. Demonstration of pseudo-ductility in high performance glass/epoxy composites by hybridisation with thin-ply carbon prepreg. Composites Part A: Applied Science and Manufacturing. 2013;52:23-30.

[3] Kretsis G. A review of the tensile, compressive, flexural and shear properties of hybrid fibre-reinforced plastics. Composites. 1987;18(1):13-23.

[4] Zhang J, Chaisombat K, He S, Wang CH. Hybrid composite laminates reinforced with glass/carbon woven fabrics for lightweight load bearing structures. Materials \& Design. 2012;36:75-80.

[5] Dai G, Mishnaevsky Jr L. Fatigue of hybrid glass/carbon composites: 3D computational studies. Composites Science and Technology. 2014;94:71-79.

[6] Hayashi T. On the improvement of mechanical properties of composites by hybrid composition. Proc 8th Intl Reinforced Plastics Conference. 1972:149-152.

[7] $\mathrm{Yu} \mathrm{H}$, Longana ML, Jalalvand M, Wisnom MR, Potter KD. Pseudo-ductility in intermingled carbon/glass hybrid composites with highly aligned discontinuous fibres. Composites Part A: Applied Science and Manufacturing. 2015;73:35-44. 
[8] Bunsell AR, Harris B. Hybrid carbon and glass fibre composites. Composites. 1974;5(4):157-164.

[9] Dong C, Davies IJ. Flexural and Tensile Moduli of Unidirectional Hybrid Epoxy Composites Reinforced by S-2 Glass and T700S Carbon Fibres. Materials \& Design. 2014;54:893-899.

[10] Dorey G, Sidey GR, Hutchings J. Impact properties of carbon fibre/Kevlar 49 fibre hybrid composites. Composites. 1978;9(1):25-32.

[11] Imieliniska K, Castaings M, Wojtyra R, Haras J, Le Clezio E, Hosten B. Air-coupled ultrasonic C-scan technique in impact response testing of carbon fibre and hybrid: glass, carbon and Kevlar/epoxy composites. Journal of Materials Processing Technology. 2004;157:513-522. [12] Guo Y, Sun Q, Wu L. Study of Dynamic Impact Behaviors and Ballistic Properties of Hybrid Composites. In: Aliabadi MH, Abela S, Baragetti S, Guagliano M, Lee HS, editors. Advances in Fracture and Damage Mechanics VIII, vol. 417-4182010. p. 213-216.

[13] Zweben C. Tensile strength of hybrid composites. Journal of Materials Science. 1977;12(7):1325-1337.

[14] Fiore V, Valenza A, Di Bella G. Mechanical behavior of carbon/flax hybrid composites for structural applications. Journal of Composite Materials. 2012;46(17):2089-2096.

[15] Peijs A, Catsman P, Govaert LE, Lemstra PJ. Hybrid composites based on polyethylene and carbon fibres Part 2: Influence of composition and adhesion level of polyethylene fibers on mechanical properties. Composites. 1990;21(6):513-521.

[16] Swolfs Y, Crauwels L, Van Breda E, Gorbatikh L, Hine P, Ward I, et al. Tensile behaviour of intralayer hybrid composites of carbon fibre and self-reinforced polypropylene. Composites Part A: Applied Science and Manufacturing. 2014;59:78-84.

[17] Manders PW, Bader MG. The strength of hybrid glass/carbon fibre composites Part 1 Failure strain enhancement and failure mode. Journal of Materials Science. 1981;16(8):22332245.

[18] Phillips LN. On the usefulness of glass-fibre-carbon hybrids. British Plastics Federation Congress, Brighton, United Kingdom1976.

[19] Swolfs Y, Gorbatikh L, Verpoest I. Fibre hybridisation in polymer composites: a review. Composites Part A: Applied Science and Manufacturing. 2014;67:181-200.

[20] Xing J, Hsiao GC, Chou TW. A dynamic explanation of the hybrid effect. Journal of Composite Materials. 1981;15(SEP):443-461.

[21] Swolfs Y, McMeeking RM, Gorbatikh L, Verpoest I. The effect of fibre dispersion on initial failure strain and cluster development in unidirectional carbon/glass hybrid composites. Composites Part A: Applied Science and Manufacturing. 2015;69:279-287.

[22] Swolfs Y, Gorbatikh L, Romanov V, Orlova S, Lomov SV, Verpoest I. Stress concentrations in an impregnated fibre bundle with random fibre packing. Composites Science and Technology. 2013;74:113-120.

[23] Swolfs Y, McMeeking RM, Verpoest I, Gorbatikh L. Matrix cracks around fibre breaks and their effect on stress redistribution and failure development in unidirectional composites. Composites Science and Technology. 2015;108:16-22.

[24] Xia ZH, Curtin WA. Multiscale modeling of damage and failure in aluminum-matrix composites. Composites Science and Technology. 2001;61(15):2247-2257.

[25] Scop PM, Argon AS. Statistical theory of strength of laminated composites II. Journal of Composite Materials. 1969;3(JAN):30-47.

[26] Blassiau S, Thionnet A, Bunsell AR. Micromechanisms of load transfer in a unidirectional carbon fibre-reinforced epoxy composite due to fibre failures. Part 1: Micromechanisms and 3D analysis of load transfer: The elastic case. Composite Structures. 2006;74(3):303-318.

[27] Blassiau S, Thionnet A, Bunsell AR. Micromechanisms of load transfer in a unidirectional carbon fibre-reinforced epoxy composite due to fibre failures. Part 2: Influence of viscoelastic 
and plastic matrices on the mechanisms of load transfer. Composite Structures. 2006;74(3):319-331.

[28] Scott AE, Sinclair I, Spearing SM, Thionnet A, Bunsell AR. Damage accumulation in a carbon/epoxy composite: Comparison between a multiscale model and computed tomography experimental results. Composites Part A: Applied Science and Manufacturing. 2012;43(9):1514-1522.

[29] Mahesh S, Phoenix SL, Beyerlein IJ. Strength distributions and size effects for 2D and 3D composites with Weibull fibers in an elastic matrix. International Journal of Fracture. 2002;115(1):41-85.

[30] Pimenta S, Pinho ST. Hierarchical scaling law for the strength of composite fibre bundles. Journal of the Mechanics and Physics of Solids. 2013;61(6):1337-1356.

[31] Thionnet A, Chou HY, Bunsell A. Fibre break processes in unidirectional composites. Composites Part A: Applied Science and Manufacturing. 2014;65:148-160.

[32] Swolfs Y, Morton H, Scott AE, Gorbatikh L, Reed PAS, Sinclair I, et al. Synchrotron radiation computed tomography for experimental validation of a tensile strength model for unidirectional fibre-reinforced composites. Composites Part A: Applied Science and Manufacturing. 2015;77:106-113.

[33] Fukuda H. An advanced theory of the strength of hybrid composites. Journal of Materials Science. 1984;19(3):974-982.

[34] Swolfs Y, Gorbatikh L, Verpoest I. Stress concentrations in hybrid unidirectional fibrereinforced composites with random fibre packings. Composites Science and Technology. 2013;85:10-16.

[35] Fukunaga H, Tsu-Wei C, Fukuda H. Strength of intermingled hybrid composites. Journal of Reinforced Plastics and Composites. 1984;3(2):145-160.

[36] Swolfs Y, Czél G, Jalalvand M, Wisnom MR, Verpoest I, Gorbatikh L. Model validation of the hybrid effect in carbon/glass hybrid composites. In: González C, López C, Llorca J, editors. 7th International Conference on Composites Testing and Model Identification, Madrid, Spain: IMDEA; 2015.

[37] Swolfs Y, Verpoest I, Gorbatikh L. Issues in strength models for unidirectional fibrereinforced composites related to Weibull distributions, fibre packings and boundary effects. Composites Science and Technology. 2015;114:42-49.

[38] Okabe T, Sekine H, Ishii K, Nishikawa M, Takeda N. Numerical method for failure simulation of unidirectional fiber-reinforced composites with spring element model. Composites Science and Technology. 2005;65(6):921-933.

[39] Okabe T, Ishii K, Nishikawa M, Takeda N. Prediction of Tensile Strength of Unidirectional CFRP Composites. Advanced Composite Materials. 2010;19(3):229-241.

[40] Xia Z, Curtin WA, Peters PWM. Multiscale modeling of failure in metal matrix composites. Acta Materialia. 2001;49(2):273-287.

[41] Melro AR, Camanho PP, Pinho ST. Generation of random distribution of fibres in longfibre reinforced composites. Composites Science and Technology. 2008;68(9):2092-2102.

[42] Romanov V, Lomov SV, Swolfs Y, Orlova S, Gorbatikh L, Verpoest I. Statistical analysis of real and simulated fibre arrangements in unidirectional composites. Composites Science and Technology. 2013;87:126-134.

[43] Thomason JL. On the application of Weibull analysis to experimentally determined single fibre strength distributions. Composites Science and Technology. 2013;77:74-80.

[44] Phoenix SL, Sexsmith RG. Clamp effects in fiber testing. Journal of Composite Materials. 1972;6(JUL):322-337.

[45] Watanabe J, Tanaka F, Okuda H, Okabe T. Tensile strength distribution of carbon fibers at short gauge lengths. Advanced Composite Materials. 2014;23(5-6):535-550.

[46] Beyerlein IJ, Phoenix SL. Statistics for the strength and size effects of microcomposites with four carbon fibers in epoxy resin. Composites Science and Technology. 1996;56(1):75-92. 
[47] Okabe T, Takeda N, Kamoshida Y, Shimizu M, Curtin WA. A 3D shear-lag model considering micro-damage and statistical strength prediction of unidirectional fiber-reinforced composites. Composites Science and Technology. 2001;61(12):1773-1787.

[48] Fitzer E. PAN-based carbon fibers - present state and trend of the technology from the viewpoint of possibilities and limits to influence and to control the fiber properties by the process parameters. Carbon. 1989;27(5):621-645.

[49] Naito K, Yang JM, Tanaka Y, Kagawa Y. The effect of gauge length on tensile strength and Weibull modulus of polyacrylonitrile (PAN)- and pitch-based carbon fibers. Journal of Materials Science. 2012;47(2):632-642. 